\title{
Evaluation of isokinetic muscle strength of upper limb and the relationship with pulmonary function and respiratory muscle strength in stable COPD patients
}

This article was published in the following Dove Press journal: International Journal of Chronic Obstructive Pulmonary Disease

\author{
Xiaodan Liu ${ }^{1,2}$ \\ Peijun $\mathrm{Li}^{3}$ \\ Zhenwei Wang ${ }^{4}$ \\ Yufan Lu (D) ${ }^{3}$ \\ Ning $\mathrm{Li}^{3}$ \\ Lu Xiao' \\ Hongxia Duan' \\ Zhengrong Wang ${ }^{3}$ \\ Jian $\mathrm{Li}^{3}$ \\ Chunlei Shan ${ }^{1,2}$ \\ Weibing $\mathrm{Wu}\left(\mathbb{1 D}^{3}\right.$
}

'School of Rehabilitation Science, Shanghai University of Traditional Chinese Medicine, Shanghai, People's Republic of China; ${ }^{2}$ Institute of Rehabilitation Medicine, Shanghai Academy of Traditional Chinese Medicine, Shanghai, People's Republic of China; ${ }^{3}$ Department of Sports Medicine, Shanghai University of Sport, Shanghai, People's Republic of China; ${ }^{4}$ Department of Respiratory Medicine, Yueyang Hospital of Integrated Traditional Chinese and Western Medicine, Shanghai, People's Republic of China
Background: Upper limb muscle strength plays an important role in respiratory and pulmonary function, and limited research focuses on the role of strength and endurance of the elbow extensor and flexor. This study was conducted to accurately assess upper limb muscle function and quantified associations with pulmonary function and respiratory muscle strength in patients with stable chronic obstructive pulmonary disease (COPD).

Methods: In this cross-sectional study, patients with stable COPD treated in Yue-Yang Integrative Medicine Hospital from March 2014 to March 2016 were recruited. All participants underwent a pulmonary function test (forced expiratory volume in first second/forced vital capacity, FEV1/FVC; percentage value of predicted FEV1, FEV1\%pred), a respiratory muscle strength test (maximal inspiratory pressure, MIP; maximal expiratory pressure, MEP), and an isokinetic test of dominant upper limb after a 24-hr interval (peak torque, $\mathrm{PT}$; PT/body weight, PT/BW; total work, TW; endurance ratio, ER).

Results: A total of 88 patients with stable COPD (age: $65.5 \pm 8.7$ years) were recruited, of which $73 \%$ (64 patients) were male. In the multiple stepwise regression analysis, sex remained as significant impactors in the final model for FEV $1 \%$ pred (adjusted $\mathrm{R}^{2}=0.243$, $P<0.001$ ). Elbow flexor PT/BW and ER, sex, and BMI remained as significant impactors in the final model for FEV1/FVC (adjusted $\mathrm{R}^{2}=0.255, P<0.01$ ). Elbow flexor TW remained as significant impactors for MIP (adjusted $\mathrm{R}^{2}=0.112, P=0.001$ ), while elbow extensor PT and $\mathrm{PT} / \mathrm{BW}$ and sex remained as significant impactors for MEP (adjusted $\mathrm{R}=0.385, P<0.01$ ).

Conclusion: In stable COPD, pulmonary function and respiratory muscle strength are associated with upper limb muscle strength. In particular, elbow flexor endurance is likely an important impactor for pulmonary function and inspiratory muscle strength, while elbow extensor strength is of importance for expiratory muscle strength.

Keywords: chronic obstructive pulmonary disease, muscle strength, endurance, elbow joint, isokinetic test

\section{Introduction}

Chronic obstructive pulmonary disease (COPD) is characterized by respiratory symptoms and skeletal muscle dysfunction. ${ }^{1}$ The interaction causes a significant decrease in the exercise capacity in patients with COPD, forming a vicious circle that accelerates disease progression. ${ }^{2}$ Epidemiological research found that COPD is expected to rise from the 11th in 2007 to the 7 th in 2020 in terms of leading to the years of life lost. ${ }^{3}$ The mortality rate of COPD is 3 million per year worldwide approximately and has become the third leading cause of death. ${ }^{4}$
School of Rehabilitation Science, Shanghai University of Traditional Chinese

Medicine, Shanghai, People's Republic of China

Email tcm4463@outlook.com

Weibing Wu

Department of Sports Medicine, Shanghai University of Sport, Shanghai, People's

Republic of China

Emailwwb75@I26.com
International Journal of Chronic Obstructive Pulmonary Disease 2019:14 2027-2036

2027

DovePress $f$ in $\boldsymbol{v}$ 
To better administrate the patients with COPD, an accurate evaluation must be applied to elucidate the condition of patients. Since respiratory symptoms are the main characteristic of COPD, providing diagnosis and treatment of the disease based on a comprehensive assessment of the respiratory system is the main content of clinical work. At present, major clinical tools for assessing respiratory function include pulmonary function tests, respiratory muscle strength tests, and questionnaires of dyspnea. Of these, the index of pulmonary function named forced expiratory volume in first second/forced vital capacity (FEV1/FVC) is the gold criterion for diagnosing the presence of an obstructive ventilatory disorder, and the percentage value of predicted FEV1 (FEV1\%pred) is a common recommendation for dividing the degree of airflow limitation. ${ }^{2}$ Respiratory muscle function often evaluated by indirect methods and expressed by the maximum spontaneous breathing pressure which is induced by muscles involved in exhalation and inhalation. ${ }^{5,6}$ Although the tests mentioned above require professional instruments and personnel to strictly conduct, the clinical prevalence and acceptance rate of these tests are still higher due to the importance in diagnosing and assessing COPD.

Skeletal muscle function plays an important role in the exercise capacity and activities of daily living and can be used to predict the mortality of COPD patients independent of lung function. ${ }^{1,7,8}$ Currently, the primary means of assessing skeletal muscle function include isometric, isotonic, and isokinetic test. The isometric test reflects the muscle strength when the joint is fixed in a certain angle and is used to evaluate the resisting capacity in the case of constant muscle length. This includes a handgrip test for the distal upper limb and dynameter test for the lower limb. ${ }^{9,10}$ Isotonic testing reflects the muscle strength during full-range motion and is used to evaluate the dynamic resisting capacity. It is often conducted in 1 repetition maximums (1RM). ${ }^{11,12}$ Isokinetic testing is used to evaluate the capacity of motion under a compliant resistance at a constant speed ${ }^{13,14}$ and can obtain the indexes of absolute muscle strength, relative muscle strength (eliminating the effects of different body weight), absolute endurance, and relative endurance (capacity of repeat contractions). Of these, isometric and isotonic tests are widely used in clinical evaluation because of the characteristics of simplicity, ease of operation, and no requirement of sophisticated instruments. However, in order to accurately determine the condition of COPD patients, accurate assessment of skeletal muscle function and its role in COPD should be emphasized.

Upper limbs are involved in a variety of daily activities, and the movement of upper limbs may interfere with the normal respiratory processes by affecting chest volume and coordinated movements of the chest and abdomen. ${ }^{15,16}$ Upper limb muscle strength, especially hand grip strength, is significantly correlated with pulmonary function in older people. ${ }^{17-19}$ The possible explanation may be that upper limb muscle strength is a surrogate for respiratory muscle strength, while respiratory muscle strength associates with pressure gradients and air exchange which impact pulmonary function. ${ }^{17}$ In addition, upper limb muscle strength shows an impact on the efficiency of ventilation, ${ }^{20}$ can impact on the exercise capacity, and then interacts with pulmonary function. Overall, increasing studies focus on the role of skeletal muscle in upper limb and found significant relationships with pulmonary function, respiratory muscle strength, exercise capacity, dyspnea, quality of life, and re-admission in COPD patients. ${ }^{12,21,22}$ For example, Toosizadeh et $\mathrm{al}^{21}$ measured the kinematics and kinetics of elbow flexion in COPD patients and found that elbow moment, speed, and power correlated well with pulmonary function (FEV1, FVC, peak expiratory flow rate). Fewer studies focused on an accurate assessment of skeletal muscle function and explored the associations of strength and endurance in extensor and flexor of upper limb with pulmonary function and respiratory muscle strength in COPD patients. Hence, this study applies isokinetic test to evaluate the function of extensors and flexors of upper limbs in COPD patients, and then investigates their associations with lung function and respiratory muscle strength. The study results can provide a reference to define which components of upper limb muscle function correlate with pulmonary function and respiratory muscle strength. The hypothesis of this study is that the function of extensor and flexor of upper limbs significantly correlates with respiratory muscle strength and pulmonary function.

\section{Methods}

\section{Participants}

This cross-sectional study was a secondary data analysis collected during the randomized controlled trials, ${ }^{23}$ investigating the effects of traditional Chinese exercise in COPD patients. The study was carried out in accordance with the Declaration of Helsinki, and ethical approval was obtained from the Ethics Committee of Yue-Yang Integrative Medicine 
Hospital, affiliated with Shanghai University of Traditional Chinese Medicine (Shanghai, China) (No. 048 (2013)). Written informed consent was obtained from all patients.

COPD patients were recruited from Yue-Yang Integrative Medicine Hospital affiliated to Shanghai University of Traditional Chinese Medicine from March 2014 to March 2016. The inclusion criteria were as follows: diagnosis with COPD according to physician assessment and spirometry $(\mathrm{FEV} 1 / \mathrm{FVC}<0.7),{ }^{24}$ age range of $40-80$, and no acute exacerbation within 4 weeks. The exclusion criteria included the presence of other pulmonary diseases like bronchial asthma, severe cardiovascular disease including but not limited to arrhythmia and coronary atherosclerotic heart disease, neuromuscular disease, or mental disease such as a cognitive impairment that could influence outcomes.

\section{Outcome measures}

Pulmonary function and respiratory muscle strength tests were conducted after collecting basic characteristics. Twenty-four hours later, the isokinetic test for upper limb skeletal muscle was conducted. Measurements used in this analysis were performed at a baseline, before initiating the rehabilitation program.

\section{Pulmonary function and respiratory muscle strength tests}

Pulmonary function and respiratory muscle strength tests were performed (MasterScreen-PFT, Jaeger, Germany) by specialized personnel in a quiet room according to the guidelines of the American Thoracic Society/European Respiratory Society (ATS/ERS). ${ }^{25}$

Before the pulmonary function test, participants were given instructions to avoid leakage of gas from the mouth. During the test, participants wore nose clips and were required to complete normal and slow breathing at least 3 times before maximum inspiration and maximum exhalation. The best value of the 3 repetitions was documented, and the 3 values varied by no more than 5\%. Parameters recorded for analysis were FEV1\%pred and FEV1/FVC after salbutamol inhalation for $15 \mathrm{~min}$. The predicted value of FEV1 was calculated by the formula embedded in the instrument (Table 1). ${ }^{23}$

The respiratory muscle strength test indirectly assesses the inspiratory muscle strength and expiratory muscle strength by the maximum inspiratory pressure (MIP) and the maximum expiratory pressure (MEP). The inspiratory muscle strength was evaluated at residual volumes and the expiratory muscle strength was evaluated at total lung capacity. According to the guidelines of ATS/ERS, ${ }^{5}$ participants were required to complete the maximal inhale and exhale duration of $3 \mathrm{~s}$. The procedure was repeated 3 times, with 1-min rest intervals, and the maximum values were recorded.

\section{Isokinetic muscle strength test}

Isokinetic dynamometer (CON-TREX, Physiomed, Germany) was used to evaluate the strength of elbow extensor and flexor muscles on the self-reported dominant side by experienced personnel based on the instruction manual. The test parameters of the isokinetic test protocol were set according to previous studies. ${ }^{26-28}$

After a warm-up exercise (gentle walking), the participant was positioned in an upright seated position with a belt applied across the chest and pelvis. The rotational axis of the dynamometer was positioned at the humeral lateral condyle, and the end resistance pad was positioned $2-3 \mathrm{~cm}$ above the wrist. Before measurement, participants were asked to determine their comfortable range of motion.

The mode of test was set as centripetal-centripetal contraction, and 2 velocities of $60 \%$ s and $180 \%$ s were selected. At the velocity of $60 \%$, participants were required to perform 5 maximal contractions in the ranged motion angles. At the velocity of $180^{\circ} \mathrm{s}$, participants were required to perform 30 maximal contractions in the ranged motion angles. Standard verbal encouragement was given to every participant. Indexes recorded for $60 \%$ s were peak torque (PT) and PT/body weight (PT/BW). The former denotes maximal muscle strength that is obtained from the 5 maximal contractions. The latter represents relative maximal muscle strength, calculated by the ratio of PT to body weight. Indexes recorded for $180^{\circ} / \mathrm{s}$ were total work (TW) and endurance ratio (ER), the former denoting muscle endurance obtained from the 30 maximal contractions, and the latter representing relative muscle endurance, calculated by the ratio of the TW of post 15 repetitions to the TW of pre 15 repetitions.

\section{Statistical analysis}

The data were analyzed using SPSS 24.0 (IBM Corp., Armonk, NY, USA). The Shapiro-Wilk test was used to test the normality of continuous variables. Normal continuous variables were presented as mean and standard deviations, and non-normal continuous variables were presented as median (interquartile range, IQR 25-75). Categorical data were presented as frequencies and percentages. Relationship of sex, BMI, duration of disease, extensor and flexor PT/BW, 
extensor and flexor TW, extensor ER with FEV1\%pred and MEP was assessed by Spearman's rank correlation coefficient. Relationship of all variables with FEV1/FVC and MIP was assessed by Spearman's rank correlation coefficient. Pearson correlations for normal distribution were used to investigate the relationship of age, extensor and flexor PT, flexor ER, with FEV1\%pred and MEP (both reported as $r$ value). The variables that showed a significant correlation with pulmonary function and respiratory muscle strength $(P<0.05)$ were included in the following multiple stepwise regression analysis to examine the deeper relationship with pulmonary function and respiratory muscle strength. Dependent variables included FEV1\%pred, FEV1/FVC, MIP, and MEP. Independent variables were parameters acquired by an isokinetic muscle test, including PT, PT/ BW, TW, and ER. Covariates included age, sex, BMI, and duration of disease. For FEV1\% \%red, extensor and flexor PT/ BW were included as independent variables in the first model, and sex was added as covariates in the second model. As for FEV1/FVC, extensor and flexor PT/BW, and flexor ER were included as independent variables in the first model, and sex and BMI were added as covariates in the second model. For MIP, extensor and flexor PT, PT/BW, TW, and flexor ER were included as independent variables in the first model, and age was added as covariates in the second model. As for MEP, extensor and flexor PT, TW, and extensor PT/BW were included as independent variables in the first model, and sex and BMI were added as covariates in the second model. A two-sided $P<0.05$ was considered as statistically significant.

\section{Results}

Characteristics of the participants are presented in Table 2. A total of 88 patients ( $73 \%$ male) with stable COPD were recruited. The age of patients was $65.5 \pm 8.7$ years, and the median duration of disease was 11.5 years (IQR 25-75, 10-14 years). Based on the severity classification of Global Initiative for Chronic Obstructive Lung Disease (GOLD), patients mostly had moderate airflow limitation with a post-bronchodilator FEV1\%pred of $57.18 \pm 17.51$. Of which, $7(8 \%)$ were mild, $50(57 \%)$ moderate, 27 $(30 \%)$ severe, and $4(5 \%)$ very severe. The patients included in this study had a BMI of $22.8 \mathrm{~kg} / \mathrm{m}^{2}$ (IQR $25-75 \square 20.3-25.3 \mathrm{~kg} / \mathrm{m}^{2}$ ), and $32(36 \%)$ of them had a BMI of $<21 \mathrm{~kg} / \mathrm{m}^{2}$ indicating a possibility of sarcopenia according to the statement of American Thoracic Society/ European Respiratory Society. ${ }^{29}$
Table I The formula to calculate the predicted value of FEVI

\begin{tabular}{|l|l|l|}
\hline Indicators & Gender & Formula \\
\hline FEVI & Male & $0.043 *$ (height*100)-0.029*age-2.49 \\
& Female & $0.0395 *$ (height*100)-0.025*age-2.6 \\
\hline
\end{tabular}

Notes: Height was expressed as meter.

Abbreviation: FEVI, forced expiratory volume in I second.

Table 2 Patient demographics

\begin{tabular}{|l|l|}
\hline Variables & Overall (n=88) \\
\hline Age, years & $65.5 \pm 8.7$ \\
Sex (M/F), $\mathrm{n}$ & $64 / 24$ \\
$\mathrm{BMI}, \mathrm{kg} / \mathrm{m}^{2}$ & $22.8(20.3-25.3)$ \\
Duration of disease, years & $11.5(10-14)$ \\
\hline Pulmonary function & \\
FEVI\%pred, \% & $57.18 \pm 17.5 \mathrm{I}$ \\
FEVI/FVC, \% & $60.58(51.24-67.39)$ \\
\hline Respiratory muscle strength & \\
MIP, kPa & $4.15(3.6-4.96)$ \\
MEP, kPa & $4.92 \pm 1.47$ \\
\hline Isokinetic muscle strength & \\
Extensor PT, Nm & $38.85 \pm 9.89$ \\
Flexor PT, Nm & $38.43 \pm 8.45$ \\
Extensor PT/BW, Nm/kg & $0.59(0.52-0.7)$ \\
Flexor PT/BW, Nm/kg & $0.59(0.51-0.69)$ \\
Extensor TW, J & $380.05(304.78-436)$ \\
Flexor TW, J & $347.5(291.23-4 \mid 5.4)$ \\
Extensor ER & $0.67(0.61-0.74)$ \\
Flexor ER & $0.7 \pm 0.12$ \\
\hline
\end{tabular}

Notes: Data are expressed in the form of mean \pm SD or median (IQR 25-75) or $n$ as appropriate.

Abbreviations: BMI, body mass index; BW, body weight; ER, endurance ratio; F, female; FEVI, forced expiratory volume in the first second; FVC, forced vital capacity; M, male; MIP, maximal inspiratory pressure; MEP, maximal expiratory pressure; PT, peak torque; TW, total work.

The results of relationships between pulmonary function and other variables are presented in Table 3. Among these, sex revealed positive $(\mathrm{r}=0.415, P<0.001)$, and extensor $\mathrm{PT} /$ $\mathrm{BW}$ and flexor PT/BW showed weak and negative $(\mathrm{r}=$ $-0.24, P=0.024$ and $\mathrm{r}=-0.249, P=0.019$, respectively) correlations with FEV1\%pred. Sex, BMI, and flexor ER demonstrated weak and positive correlations ( $\mathrm{r}=0.224$ to 0.287, $P<0.05$ ), while extensor PT/BW and flexor PT/BW showed weak and negative correlations $(\mathrm{r}=-0.23, P=0.031$, and $\mathrm{r}=-0.435, P<0.001$, respectively) with FEV1/FVC. In the final model of the multiple stepwise regression analysis for FEV1\%pred, only sex remained as significant impactors with an adjusted $\mathrm{R}^{2}$ of 0.243 (Table 4). Moreover, males had $19.609 \%$ higher of FEV1\%pred than females. Therefore, 
Table 3 Relationship between pulmonary function and other variables

\begin{tabular}{|l|l|l|l|l|}
\hline \multirow{2}{*}{ Variables } & \multicolumn{2}{|l|}{ FEV I\%pred, \% } & \multicolumn{2}{l|}{ FEVI/FVC, \% } \\
\cline { 2 - 5 } & $\mathbf{r}$ & P-value & $\mathbf{r}$ & P-value \\
\hline Age, years & -0.07 & $0.5 \mathrm{I}$ & 0.021 & 0.845 \\
Sex, M/F & 0.505 & $<0.00 \mathrm{I}$ & 0.35 & $0.00 \mathrm{I}$ \\
$\mathrm{BMI}, \mathrm{kg} / \mathrm{kg}^{2}$ & 0.188 & 0.079 & 0.224 & 0.036 \\
Duration, years & $-0.07 \mathrm{I}$ & 0.513 & 0.139 & 0.195 \\
MIP, kPa & 0.06 & 0.58 & 0.134 & 0.215 \\
MEP, kPa & -0.175 & 0.102 & 0.02 & 0.854 \\
Extensor PT, Nm & -0.153 & 0.155 & -0.005 & 0.966 \\
Flexor PT, Nm & -0.073 & 0.502 & -0.176 & 0.101 \\
Extensor PT/BW, Nm/kg & -0.24 & 0.024 & -0.23 & 0.031 \\
Flexor PT/BW, Nm/kg & -0.249 & 0.019 & -0.435 & $<0.001$ \\
Extensor TW, J & -0.048 & 0.655 & 0.101 & 0.35 \\
Flexor TW, J & -0.08 & 0.45 & $-0.05 \mathrm{I}$ & 0.635 \\
Extensor ER & 0.145 & 0.179 & 0.183 & 0.088 \\
Flexor ER & 0.107 & 0.321 & 0.232 & 0.029 \\
\hline
\end{tabular}

Notes: Relationships were calculated by Pearson or Spearman coefficients analysis as appropriate. The degree of significance was set at $P<0.05$.

Abbreviations: BMI, body mass index; BW, body weight; ER, endurance ratio; FEVI, forced expiratory volume in the first second; FVC, forced vital capacity; MIP, maximal inspiratory pressure; MEP, maximal expiratory pressure; PT, peak torque; TW, total work.

we stratified these analyses on sex. In the sensitivity analysis, results showed no substantial difference from the primary analysis and are not reported here. With respect to FEV1/FVC, Flexor PT/BW and ER remained as significant impactors in the first model with an adjusted $\mathrm{R}^{2}$ of 0.161 (Table 5). In the final model for FEV1/FVC, Flexor PT/BW and ER, sex, and BMI remained as impactors with an adjusted $\mathrm{R}^{2}$ of 0.255 , although the flexor PT/BW was no longer significant (Table 6).

The results of relationships between respiratory muscle strength and other variables are presented in Table 7. Among these, age, extensor and flexor PT, PT/BW, TW, and flexor ER revealed weak to moderate and positive correlations with MIP ( $\mathrm{r}=0.217$ to $0.471, P<0.05$ ). Sex showed weak and negative correlation $(\mathrm{r}=-0.389$, $P<0.001$ ), and BMI, extensor and flexor PT, TW, and extensor PT/BW revealed weak to moderate and positive correlations with MEP ( $\mathrm{r}=0.225$ to $0.558, P<0.05$ ). In the final model of the multiple stepwise regression analysis for MIP, only flexor TW remained as significant impactors with an adjusted $\mathrm{R}^{2}$ of 0.112 (Table 8 ). With respect to MEP, extensor PT and PT/BW remained as significant impactors in the first model with an adjusted $\mathrm{R}^{2}$ of 0.333 (Table 9), and extensor PT, PT/BW, and sex remained as significant impactors in the final model with an adjusted $\mathrm{R}^{2}$ of 0.385 (Table 10).

\section{Discussion}

Results of this study revealed that isokinetic muscle strength of upper limb significantly correlates with pulmonary function and respiratory muscle strength in stable COPD patients. In detail, relative muscle strength and endurance of elbow flexor showed significant relationships with FEV1/FVC, absolute endurance of the elbow flexor

Table 4 Results of multivariate stepwise regression analysis of the FEVI\%pred and the independent determinants taking into account covariates

\begin{tabular}{|l|l|l|l|l|}
\hline Variables & Coefficient $(95 \%$ CI) & Standardized $\boldsymbol{\beta}$ & $P$-value \\
\hline Intercept & $32.224(22.45 \mathrm{I}-41.997)$ & & \\
Sex & $19.609(12.36 \mathrm{I}-26.857)$ & 0.502 & $<0.00 \mathrm{I}$ & $\mathrm{I}$ \\
Adjusted $\mathrm{R}^{2}=0.243$ & & & & \\
\hline
\end{tabular}

Notes: The degree of significance was set at $P<0.05$. Independent determinants: Extensor PT/BW, Flexor PT/BW. Covariates: sex.

Abbreviations: $\beta$, regression coefficient; $\mathrm{BW}$, body weight; $\mathrm{Cl}$, confidence interval; FEVI, forced expiratory volume in the first second; PT, peak torque.

Table 5 Results of multivariate stepwise regression analysis of the FEVI/FVC and the independent determinants

\begin{tabular}{|l|l|l|l|l|}
\hline Variables & Coefficient $(\mathbf{9 5 \%} \mathbf{C l})$ & Standardized $\boldsymbol{\beta}$ & $\boldsymbol{P}$-value \\
\hline Intercept & $57.443(37.417-77.469)$ & & & \\
Flexor PT/BW & $-36.6(-57.919--15.281)$ & -0.336 & 0.001 & \\
Flexor ER & $34.015(10.891-57.14)$ & 0.288 & 0.004 & 1.007 \\
Adjusted $R^{2}=0.161$ & & & & 1.007 \\
\hline
\end{tabular}

Notes: The degree of significance was set at $P<0.05$. Independent determinants: Extensor PT/BW, Flexor PT/BW, Flexor ER.

Abbreviations: $\beta$, regression coefficient; $\mathrm{BW}$, body weight; $\mathrm{Cl}$, confidence interval; ER, endurance ratio; FEVI, forced expiratory volume in the first second; FVC, forced vital capacity; PT, peak torque. 
Table 6 Results of multivariate stepwise regression analysis of the FEVI/FVC and the independent determinants taking into account covariates

\begin{tabular}{|l|l|l|l|l|}
\hline Variables & Coefficient $\mathbf{( 9 5 \% ~ C l )}$ & Standardized $\boldsymbol{\beta}$ & $P$-value \\
\hline Intercept & $12.184(-20.67-45.038)$ & & & \\
Flexor PT/BW & $-19.19(-41.514-3.134)$ & -0.176 & $0.09 I$ & 1.243 \\
Flexor ER & $35.988(14.128-57.847)$ & 0.305 & 0.002 & 1.013 \\
Sex & $8.946(2.924-14.968)$ & 0.292 & 0.004 & 1.14 \\
BMI & $0.951(0.142-1.76)$ & 0.228 & 0.022 & 1.109 \\
Adjusted $R^{2}=0.255$ & & & & \\
\hline
\end{tabular}

Notes: The degree of significance was set at $P<0.05$. Independent determinants: Extensor PT/BW, Flexor PT/BW, Flexor ER. Covariates: sex, BMI.

Abbreviations: $\beta$, regression coefficient; $\mathrm{BMI}$, body mass index; $\mathrm{BW}$, body weight; $\mathrm{Cl}$, confidence interval; $\mathrm{ER}$, endurance ratio; $\mathrm{FEVI}$, forced expiratory volume in the first second; FVC, forced vital capacity; PT, peak torque.

Table 7 Relationship between respiratory muscle strength and other variables

\begin{tabular}{|l|l|l|l|l|}
\hline \multirow{2}{*}{ Variables } & \multicolumn{2}{|l|}{ MIP, kPa } & \multicolumn{2}{l|}{ MEP, kPa } \\
\cline { 2 - 5 } & $\mathbf{r}$ & P-value & $\mathbf{r}$ & P-value \\
\hline Age, years & 0.297 & 0.005 & -0.13 & 0.224 \\
Sex, M/F & -0.173 & 0.05 & -0.389 & $<0.001$ \\
BMl, kg/kg & 0.099 & 0.358 & 0.338 & 0.001 \\
Duration, years & -0.107 & 0.32 & -0.028 & 0.793 \\
Extensor PT, Nm & 0.427 & $<0.001$ & 0.558 & $<0.001$ \\
Flexor PT, Nm & 0.361 & 0.001 & 0.421 & $<0.001$ \\
Extensor PT/BW, Nm/kg & 0.301 & 0.004 & 0.225 & 0.035 \\
Flexor PT/BW, Nm/kg & 0.217 & 0.042 & 0.07 & 0.517 \\
Extensor TW, J & 0.427 & $<0.001$ & 0.352 & 0.001 \\
Flexor TW, J & 0.471 & $<0.001$ & 0.285 & 0.007 \\
Extensor ER & 0.198 & 0.065 & -0.158 & 0.141 \\
Flexor ER & 0.26 & 0.014 & 0.039 & 0.716 \\
\hline
\end{tabular}

Notes: Relationships were calculated by Pearson or Spearman or Kendall's tau-b coefficients analysis as appropriate. The degree of significance was set at $P<0.05$. Abbreviations: BMI, body mass index; BW, body weight; ER, endurance ratio; MIP, maximal inspiratory pressure; MEP, maximal expiratory pressure; PT, peak torque; TW, total work.

correlated with MIP, and muscle strength of the elbow extensor correlated with MEP.

Isokinetic muscle strength test provides compliant resistance for certain muscle groups to evaluate the dynamic strength and endurance. During the test, muscle strength and length increased accompanied with increased muscle tension and torque output. ${ }^{13}$ The test is commonly used to evaluate the efficacy of exercise rehabilitation, and the apparatus includes CON-TREX, Biodex, and Cybex, all with superior test-retest reliability, safety, and accuracy. ${ }^{14,30}$ Previous studies found that the peripheral skeletal muscle function of COPD patients, especially in the lower limb, was significantly lower than that of healthy people. $^{26,31-33}$ Decreased skeletal muscle function is significantly associated with endurance exercise capacity, quality of life, and dyspnea and is an independent risk factor for accelerating disease progression and predicting mortality in COPD patients. ${ }^{29,34,35}$ Skeletal muscles in the upper limb including proximal muscles around the shoulder and distal muscles around the elbow are accessory respiratory muscles and play an important role in the process of respiration, especially when primary respiratory muscles occur dysfunction or cannot meet the ventilatory demand. ${ }^{11}$ Skeletal muscle function of upper limbs plays an important role in submaximal exercise tolerance of COPD patients; ${ }^{11}$ exercise capacity has interaction with pulmonary function, ${ }^{36}$ so speculation raised that skeletal muscle function of upper limbs may be associated with pulmonary function. In addition, upper limb activity participates in $80 \%$ activity of daily life, affecting the capacity

Table 8 Results of multivariate stepwise regression analysis of the MIP and the independent determinants taking into account covariates

\begin{tabular}{|l|l|l|l|l|}
\hline Variables & Coefficient (95\% Cl) & Standardized $\boldsymbol{\beta}$ & $\boldsymbol{P}$-value \\
\hline Intercept & $2.852(1.938-3.766)$ & & \\
Flexor TW, J & $0.004(0.002-0.007)$ & 0.349 & 0.001 & \\
Adjusted $\mathrm{R}^{2}=0.112$ & & & & 1 \\
\hline
\end{tabular}

Notes: The degree of significance was set at $P<0.05$. Independent determinants: Extensor PT, Flexor PT, Extensor PT/BW, Flexor PT/BW, Extensor TW, Flexor TW, Flexor ER. Covariates: age.

Abbreviations: $\beta$, regression coefficient; $\mathrm{BW}$, body weight; $\mathrm{Cl}$, confidence interval; ER, endurance ratio; MIP, maximal inspiratory pressure; PT, peak torque; TW, total work. 
Table 9 Results of multivariate stepwise regression analysis of the MEP and the independent determinants

\begin{tabular}{|l|l|l|l|}
\hline Variables & Coefficient (95\% CI) & VIF & $\boldsymbol{P}$-value \\
\hline Intercept & $2.324(1.137-3.5 I I)$ & & \\
Extensor PT, Nm & $0.117(0.077-0.156)$ & 0.782 & $<0.001$ \\
Extensor PT/BW, Nm/kg & $-3.163(-6.015--0.312)$ & -0.296 & 0.033 \\
Adjusted $\mathrm{R}^{2}=0.333$ & & & 2.352 \\
\hline
\end{tabular}

Notes: The degree of significance was set at $P<0.05$. Independent determinants: Extensor PT, Flexor PT, Extensor PT/BW, Extensor TW, Flexor TW.

Abbreviations: $\beta$, regression coefficient; $\mathrm{BW}$, body weight; $\mathrm{Cl}$, confidence interval; MEP, maximal expiratory pressure; PT, peak torque; TW, total work.

Table 10 Results of multivariate stepwise regression analysis of the MEP and the independent determinants taking into account covariates

\begin{tabular}{|c|c|c|c|c|}
\hline Variables & Coefficient $(95 \% \mathrm{CI})$ & Standardized $\beta$ & $P$-value & VIF \\
\hline Intercept & $4.183(2.463-5.902)$ & & & \\
\hline Extensor PT, Nm & $0.095(0.054-0.136)$ & 0.64 & $<0.001$ & 2.699 \\
\hline Extensor PT/BW, Nm/kg ${ }^{2}$ & $-2.981(-5.722--0.24)$ & -0.279 & 0.033 & 2.357 \\
\hline Sex & $-0.902(-1.526-0.277)$ & -0.274 & 0.005 & 1.289 \\
\hline Adjusted $R^{2}=0.385$ & & & & \\
\hline
\end{tabular}

Notes: The degree of significance was set at $\mathrm{P}<0.05$. Independent determinants: Extensor PT, Flexor PT, Extensor PT/BW, Extensor TW, Flexor TW. Covariates: sex, BMI. Abbreviations: $\beta$, regression coefficient; BMI, body mass index; BW, body weight; $\mathrm{Cl}$, confidence interval; MEP, maximal expiratory pressure; PT, peak torque; TW, total work.

of physical activity and quality of life in COPD patients. ${ }^{12}$ Tests including 1RM, handgrip, and functional exercise tests were used to evaluate the skeletal muscle function of the upper limb, which significantly correlated with endurance exercise capacity, quality of life, and dyspnea of COPD patients. ${ }^{11,12,21}$ Different test methods may explain the different results referring to the relationship with pulmonary function and respiratory muscle strength. Actually, few studies make use of the isokinetic test for evaluating of upper limb muscle function and investigate the possible role in pulmonary function and respiratory muscle strength.

In this study, weak and negative correlations between relative muscle strength of elbow extensor and flexor with FEV1\%pred were observed. This is inconsistent with a previous study which found that proximal muscle strength tested by 1RM and distal muscle strength tested by handgrip were unrelated with pulmonary function. ${ }^{12}$ This is also contrary to the moderate and positive correlations between elbow movement (include power, moment, and speed reduction) and FEV1\%pred. ${ }^{21}$ It can be speculated that the relative muscle strength in this study was adjusted by body weight, while muscle strength mentioned above was original. Results of multiple stepwise regression analysis revealed that only sex remained as a significant impactor for FEV1\%pred in COPD patients. After stratified by sex, the results were not substantially different from previous results and are not reported here. For FEV1/FVC, relative muscle strength and endurance of elbow flexor remained as impactors in this study. The relative muscle strength and endurance of elbow flexor can be considered as factors that interacted with the pulmonary function of COPD, which is consistent with results indicating that physical inactivity will accelerate the progress of COPD. ${ }^{37}$

Approximately $40-60 \%$ of the COPD patients demonstrate decreased respiratory muscle strength, and increased activity and fatigue resistance of muscle. ${ }^{38}$ Although respiratory muscle function is relatively preserved, the main complaint of COPD patients is dyspnea. ${ }^{39}$ The contradiction between respiratory muscle function and symptoms may be due to increased respiratory burden in COPD patients, resulting in a compensatory increase in respiratory activity, whereas abnormal thoracic morphology and abdominal wall tension cause abnormal muscle fiber length and tension, leading to the impairment of strengthgenerating capacity. ${ }^{38}$ Similar to the condition of respiratory muscles, upper limb muscle strength in COPD patients was significantly reduced, while the exercise capacity of the upper limbs was preserved; it is speculated that the daily recruitment of upper limb muscles to support ventilation as a continuous and submaximal activity leads to a maintained or even increased endurance. ${ }^{40}$ Moreover, 
hyperventilation was seen during upper limb exercise in COPD patients. ${ }^{41}$ This may be explained by the weakened effect of upper limb muscles in assisting respiration or the disruption of normal abdominal breathing patterns during upper limb movement. In total, skeletal muscle of upper limb plays an important role in respiratory of COPD patients. A previous study demonstrated that the speed, power, rise time, and core of upper-extremity function test were associated with MIP ( $\mathrm{r}=0.35$ to $0.69, P<0.05$ ), and the moment, speed reduction, flexion number of upperextremity function test were associated with MEP ( $\mathrm{r}=$ -0.42 to $0.42, P<0.05){ }^{21}$ Consistent with that, in this study, most of the parameters in the isokinetic test were correlated with MIP and MEP ( $\mathrm{r}=0.217$ to $0.558, P<0.05$ ), and endurance of elbow flexor was correlated with inspiratory muscle strength, muscle strength of elbow extensor was correlated with expiratory muscle strength.

The main limitation of this study is the small sample size and lack of control. Therefore, further research with a large sample size and control group should be considered to determine the impact of upper limb skeletal muscle. Moreover, this study mainly aimed to explore the potential relationship between upper limb muscle function and pulmonary function, respiratory muscle strength in COPD patients; the cause-and-effect relationship could not be determined. In this context, the relationship between upper limb muscle function and pulmonary function, respiratory muscle strength may be an interaction. Longitudinal study should be designed to investigate the cause-and-effect relationship. In addition, the variable of the FEV1 was expressed as \%pred, while other variables of isokinetic indexes were expressed in absolute values, the mixed expression may induce some interactions with anthropometric variables. Finally, this study did not correct for important variables, including comorbidities, medication, smoking, and physical activity. Therefore, the interpretation of the results should be considered very cautiously, and future studies should be designed more comprehensively.

\section{Conclusion}

Isokinetic muscle strength of flexor and extensor of upper limb correlates with pulmonary function and respiratory muscle strength in stable COPD patients. Endurance of elbow flexor plays an important role in pulmonary function and inspiratory muscle strength, while muscle strength of the elbow extensor is of important for expiratory muscle strength.

\section{Acknowledgment}

This study was supported by the national fitness project of General Administration of Sport of China (No. 2017B021), the key basic research grants from Science and Technology Commission of Shanghai Municipality (No. 16JC1400500), the directed research grants from Science and Technology Commission of Shanghai Municipality (No. 18DZ1200600), and National Natural Science Foundation of China (No. 81472163).

\section{Author contributions}

All authors contributed to data analysis, drafting and revising the article, gave final approval of the version to be published, and agree to be accountable for all aspects of the work.

\section{Disclosure}

The authors report no conflicts of interest in this work.

\section{References}

1. Rabinovich R, Vilaro J. Structural and functional changes of peripheral muscles in chronic obstructive pulmonary disease patients. Curr Opin Pulm Med. 2010;16(2):123-133. doi:10.1097/MCP.0b013e328336 $438 \mathrm{~d}$

2. Global Initiative for Chronic Obstructive Lung Disease. Global strategy for the diagnosis, management, and prevention of chronic obstructive pulmonary disease (2018 Report) Available from: http:// goldcopd.org.2018. Accessed February 5, 2018.

3. WHO. World Health Statistics 2017: Monitoring Health for the SDGs, Sustainable Development Goals. Available from: http://www.who.int/ gho/publications/world_health_statistics/en/ Accessed November 22, 2017.

4. Rabe KF, Watz H. Chronic obstructive pulmonary disease. Lancet. 2017; 389(10082):1931-1940. doi:10.1016/S0140-6736(17)31222-9.

5. Gibson GJ, Whitelaw W, Siafakas N, et al. ATS/ERS statement on respiratory muscle testing. Am J Respir Crit Care Med. 2002;166 (4):518-624. doi:10.1164/rccm.166.4.518

6. Charususin N, Dacha S, Gosselink R, et al. Respiratory muscle function and exercise limitation in patients with chronic obstructive pulmonary disease: a review. Expert Rev Respir Med. 2018;12(1):6779. doi:10.1080/17476348.2018.1398084

7. Cielen N, Maes K, Gayan-Ramirez G. Musculoskeletal disorders in chronic obstructive pulmonary disease. Biomed Res Int. 2014;2014:96 5764. doi: $10.1155 / 2014 / 965764$

8. Yoshimura K, Sato S, Muro S, et al. Interdependence of physical inactivity, loss of muscle mass and low dietary intake: Extrapulmonary manifestations in older chronic obstructive pulmonary disease patients. Geriatr Gerontol Int. 2018;18(1):88-94. doi:10.1111/ggi.13146

9. Gea J, Casadevall C, Pascual S, Orozco-Levi M, Barreiro E. Clinical management of chronic obstructive pulmonary disease patients with muscle dysfunction. $J$ Thorac Dis. 2016;8(11):3379-3400. doi:10.21037/ jtd.2016.11.105

10. Cronin J, Lawton T, Harris N, Kilding A, McMaster DT. A brief review of handgrip strength and sport performance. J Strength Cond Res. 2017;31(11):3187-3217. doi:10.1519/JSC.0000000000002149 
11. Dourado VZ, Antunes LC, Tanni SE, de Paiva SA, Padovani CR, Godoy I. Relationship of upper-limb and thoracic muscle strength to 6-min walk distance in COPD patients. Chest. 2006;129(3):551-557. doi:10.1378/ chest.129.3.551

12. Kaymaz D, Candemir İÇ, Ergün P, Demir N, Taşdemir F, Demir P. Relation between upper-limb muscle strength with exercise capacity, quality of life, and dyspnea in patients with severe chronic obstructive pulmonary disease. Clin Respir J. 2018;12(3):1257-1263. doi:10.1111/ crj. 12659

13. Chen Y, Niu M, Zhang X, Qian H, Xie A, Wang X. Effects of homebased lower limb resistance training on muscle strength and functional status in stable COPD patients. J Clin Nurs. 2017;27(5-6) e1022-e1037. doi:10.1111/jocn.14131

14. Maffiuletti N, Bizzini M, Desbrosses K, Babault N, Munzinger U. Reliability of knee extension and flexion measurements using the Con-Trex isokinetic dynamometer. Clin Physiol Funct Imaging. 2010;27(6):346-353. doi:10.1111/j.1475-097X.2007.00758.x

15. Spruit MA, Singh SJ, Garvey C, et al. An official American Thoracic Society/European Respiratory Society statement: key concepts and advances in pulmonary rehabilitation. Am J Respir Crit Care Med. 2013;188(8):e13. doi:10.1164/rccm.201309-1634ST

16. Dolmage TE, Janaudis-Ferreira T, Hill K, Price S, Brooks D, Goldstein RS. Arm elevation and coordinated breathing strategies in patients with COPD. Chest. 2013;144(1):128-135. doi:10.1378/ chest.12-2467

17. Buchman AS, Boyle PA, Wilson RS, Gu L, Bienias JL, Bennett DA. Pulmonary function, muscle strength and mortality in old age. Mech Ageing Dev. 2008;129(11):625-631. doi:10.1016/j.mad.2008.07.003

18. Sillanpaa E, Stenroth L, Bijlsma AY, et al. Associations between muscle strength, spirometric pulmonary function and mobility in healthy older adults. Age (Dordr). 2014;36(4):9667. doi:10.1007/s11357-014-9667-7

19. Son DH, Yoo JW, Cho MR, Lee YJ. Relationship between handgrip strength and pulmonary function in apparently healthy older women. $J$ Am Geriatr Soc. 2018;66(7):1367-1371. doi:10.1111/jgs.15410

20. Muller Pde T, Viegas CA, Patusco LA. Muscle strength as a determinant of oxygen uptake efficiency and maximal metabolic response in patients with mild-to-moderate COPD. J Bras Pneumol. 2012;38(5):541-549. doi:10.1590/s1806-37132012000500002

21. Toosizadeh N, Berry C, Bime C, Najafi B, Kraft M, Mohler J. Assessing upper-extremity motion: An innovative method to quantify functional capacity in patients with chronic obstructive pulmonary disease. PLoS One. 2017;12(2):e0172766. doi:10.1371/journal.pone.0172766

22. Ehsani H, Mohler MJ, Golden T, Toosizadeh N. Upper-extremity function prospectively predicts adverse discharge and all-cause COPD readmissions: a pilot study. Int J Chron Obstruct Pulmon Dis. 2019;14:39-49. doi:10.2147/COPD.S182802

23. Wu W, Liu X, Li P, Li N, Wang Z. Effect of Liuzijue exercise combined with elastic band resistance exercise on patients with COPD: a randomized controlled trial. Evid Based Complement Alternat Med. 2018;2018:2361962. doi:10.1155/2018/9567061

24. Global Initiative for Chronic Obstructive Lung Disease: Pocket guide to COPD diagnosis, management, and prevention. Available from: http:// goldcopd.org.2013. Accessed March 15, 2013.

25. Miller M, Hankinson J, Brusasco V, et al. Standardisation of spirometry "ATS/ERS task force: standardisation of lung function testing". Eur Respir J. 2005;26(2):319-338. doi:10.1183/09031936.05.00034805

26. Malaguti C, Napolis L, Villaça D, Neder J, Nery L, Dal Corso S. Relationship between peripheral muscle structure and function in patients with chronic obstructive pulmonary disease with different nutritional status. J Strength Cond Res. 2011;25(7):1795-1803. doi:10.1519/ JSC.0b013e3181e501c1
27. Butcher SJ, Pikaluk BJ, Chura RL, Walkner MJ, Farthing JP, Marciniuk DD. Associations between isokinetic muscle strength, high-level functional performance, and physiological parameters in patients with chronic obstructive pulmonary disease. Int $J$ Chron Obstruct Pulmon Dis. 2012;7:537-542. doi:10.2147/COPD.S34170

28. Wittstein J, Queen R, Abbey A, Moorman CT 3rd. Isokinetic testing of biceps strength and endurance in dominant versus nondominant upper extremities. J Shoulder Elbow Surg. 2010;19(6):874-877. doi:10.1016/j.jse.2010.01.018

29. Maltais F, Decramer M, Casaburi R, et al. An official American Thoracic Society/European Respiratory Society statement: update on limb muscle dysfunction in chronic obstructive pulmonary disease. Am J Respir Crit Care Med. 2014;189(9):e15-e62. doi:10.1164/ rccm.201402-0373ST

30. Robles PG, Mathur S, Janaudisfereira T, Dolmage TE, Goldstein RS, Brooks D. Measurement of peripheral muscle strength in individuals with chronic obstructive pulmonary disease: a systematic review. $J$ Cardiopulm Rehabil Prev. 2011;31(1):11-24. doi:10.1097/HCR.0b0 13e3181ebf302

31. Castagna O, Boussuges A, Vallier J, Prefaut C, Brisswalter J. Is impairment similar between arm and leg cranking exercise in COPD patients? Respir Med. 2007;101(3):547-553. doi:10.1016/j. rmed.2006.06.019

32. Coratella G, Rinaldo N, Schena F. Quadriceps concentric-eccentric force and muscle architecture in COPD patients vs healthy men. Hum Mov Sci. 2018;59:88-95. doi:10.1016/j.humov.2018.03.015

33. Ribeiro F, Oueslati F, Saey D, et al. Cardiorespiratory and muscle oxygenation responses to isokinetic exercise in chronic obstructive pulmonary disease. Med Sci Sports Exerc. 2019;51(5):841-849. doi:10.1249/MSS.0000000000001856

34. Hernández M, Zambom-Ferraresi F, Cebollero P, Hueto J, Cascante JA, Antón MM. The relationships between muscle power and physical activity in older men with chronic obstructive pulmonary disease. J Aging Phys Act. 2017;25(3):360-366. doi:10.1123/japa.2016-0144

35. Nyberg A, Tornberg A, Wadell K. Correlation between limb muscle endurance, strength, and functional capacity in people with chronic obstructive pulmonary disease. Physiother Can. 2016;68(1):46-53. doi:10.3138/ptc.2014-93

36. Zeng GS, Chen LC, Fan HZ, et al. The relationship between steps of 6MWT and COPD severity: a cross-sectional study. Int J Chron Obstruct Pulmon Dis. 2019;14:141-148. doi:10.2147/COPD.S188994

37. Garcia-Aymerich J, Lange P, Benet M, Schnohr P, Antó JM. Regular physical activity modifies smoking-related lung function decline and reduces risk of chronic obstructive pulmonary disease: a populationbased cohort study. Am J Respir Crit Care Med. 2007;175(5):458463. doi:10.1164/rccm.200607-896OC

38. Caron MA, Debigaré R, Dekhuijzen PN, Maltais F. Comparative assessment of the quadriceps and the diaphragm in patients with COPD. J Appl Physiol (1985). 2009;107(3):952-961. doi:10.1152/ japplphysiol.00194.2009

39. O'Donnell DE. Hyperinflation, dyspnea, and exercise intolerance in chronic obstructive pulmonary disease. Proc Am Thorac Soc. 2006;3 (2):180-184. doi:10.1513/pats.200508-093DO

40. Clark CJ, Cochrane LM, Mackay E, Paton B. Skeletal muscle strength and endurance in patients with mild COPD and the effects of weight training. Eur Respir J. 2000;15(1):92-97.

41. Gigliotti F, Coli C, Bianchi R, et al. Arm exercise and hyperinflation in patients with COPD: effect of arm training. Chest. 2005;128 (3):1225-1232. doi:10.1378/chest.128.3.1225 


\section{Publish your work in this journal}

The International Journal of COPD is an international, peer-reviewed journal of therapeutics and pharmacology focusing on concise rapid reporting of clinical studies and reviews in COPD. Special focus is given to the pathophysiological processes underlying the disease, intervention programs, patient focused education, and self management protocols. This journal is indexed on PubMed Central, MedLine and CAS. The manuscript management system is completely online and includes a very quick and fair peer-review system, which is all easy to use. Visit http://www.dovepress.com/testimonials.php to read real quotes from published authors. 